\title{
TITLE:
}

\section{Kaluza-Klein mediated supersymmetry breaking}

$\operatorname{AUTHOR}(\mathrm{S})$ :

Kobayashi, T; Yoshioka, K

\section{CITATION:}

Kobayashi, T ...[et al]. Kaluza-Klein mediated supersymmetry breaking. PHYSICAL REVIEW LETTERS 2000, 85(26): 5527-5530

ISSUE DATE:

2000-12-25

URL:

http://hdl.handle.net/2433/50541

RIGHT:

Copyright 2000 American Physical Society 


\title{
Kaluza-Klein Mediated Supersymmetry Breaking
}

\author{
Tatsuo Kobayashi ${ }^{1}$ and Koichi Yoshioka ${ }^{2}$ \\ ${ }^{1}$ Department of Physics, Kyoto University, Kyoto 606-8502, Japan \\ ${ }^{2}$ Yukawa Institute for Theoretical Physics, Kyoto University, Kyoto 606-8502, Japan
}

(Received 28 August 2000)

\begin{abstract}
The four-dimensional effective theories with compactified extra dimensions are considered. We there discuss a new framework for communicating supersymmetry breaking to the visible sector where the radius modulus, which determines the size of extra dimensions, has an auxiliary vacuum expectation value. The modulus couplings generate mass splitting in Kaluza-Klein supermultiplets and they act as messengers of supersymmetry breaking. The soft masses are expressed in terms of renormalization-group functions and the sparticle spectrum is determined by what kind of field propagates in the bulk.
\end{abstract}

PACS numbers: 12.60.Jv, 11.10.Kk, 11.25.Mj

Low-energy supersymmetry (SUSY) provides an attractive candidate for the fundamental theory beyond the standard model (SM). However, supersymmetry must be broken due to the absence of experimental signatures below the electroweak scale. So far, various scenarios for supersymmetry breaking have been proposed, and for the scenarios to be viable, several phenomenological constraints should be satisfied. For example, we must have suppressions of new flavor-changing operators which are generally introduced by superpartners of the SM fields. This fact restricts possible forms of SUSY-breaking terms [1] and SUSY-breaking mechanisms at high energy. In particular, that may require there are no direct couplings between the visible and SUSY-breaking sectors. In recently proposed scenarios [2,3] with extra dimensions, the SUSY-breaking sector is spatially separated from the visible one, and the flavor-changing operators are strongly suppressed. Another desirable property of SUSY-breaking scenarios is that the sparticle spectrum is distinctive of the scenario and detectable in future experiments. For this issue, renormalization-group effects between the SUSYbreaking and low-energy scales would also be important. In this Letter, we present a new mediation mechanism of SUSY breaking in the model with extra spatial dimensions. (For recent work for other mechanisms with extra dimensions, see Refs. [4].) As will be seen below, in our scenario, the modulus which does not directly couple to the visible sector has a nonvanishing $F$ term, and, moreover, characteristic spectra can be obtained depending on how to construct four-dimensional low-energy effective theories.

We consider four-dimensional effective theories with additional dimensions compactified at the scale $M_{c}$, which is smaller than the cutoff $\Lambda$ of the effective theories. We assume for simplicity that all the compactified dimensions have equal radius. The compactification scale, i.e., the size of extra dimensions, is determined by the vacuum expectation value of a radius modulus $T$. We here assume that in the low-energy effective theories, the modulus superfield has an auxiliary vacuum expectation value, $\langle T\rangle=M_{c}+\theta^{2} F_{T}$. In this Letter, we study the case where the contribution of the radius modulus dominates SUSY breaking. With other sources of SUSY breaking (nonzero $F$ terms), there will exist additional effects on the soft terms. When the SM fields propagate in the bulk, the corresponding massive towers of Kaluza-Klein (KK) modes appear in the four-dimensional effective theories. The mass couplings of chiral supermultiplets for the $n$th KK mode are given by the radius modulus through the superpotential

$$
W \sim n T \Phi^{(n)} \bar{\Phi}^{(n)} .
$$

Here $\bar{\Phi}$ is the SUSY-partner multiplet of $\Phi$ at each KK level. This interaction induces mass splitting between the bosonic and fermionic components of $\Phi$ and $\bar{\Phi}$, proportional to the SUSY-breaking effect $F_{T}$. For KK vector multiplets, the mass splitting in the multiplets comes from Kähler terms $\sim \int d \theta^{2} d \bar{\theta}^{2}|n T|^{2} V^{(n)} V^{(n)}$. The KK excited modes thus work as messenger fields which transmit the SUSY-breaking effect to the visible sector. In this scenario, the visible sector consists of KK zero modes and the fields confined on a "3-brane." It is noted that before Weyl rescaling there are no (nonderivative) direct couplings of the radius modulus $T$ to the visible sector. (This issue is analyzed in minimal five-dimensional supergravity [5].) This is because $T$ can couple only to the higherdimensional components of the energy-momentum tensor and the wave functions of massless fields do not depend on the extradimensional coordinates. As a result, the soft SUSY-breaking terms are generated at a quantum level in the four-dimensional effective theory. That ensures the SUSY-breaking sector is hidden and separated from our visible sector.

We derive the expressions of soft SUSY-breaking terms. Let us first consider the case where gauge fields propagate in the bulk, whereas other fields are confined on the 3-brane. The effective theory on the brane contains the KK gauge and gaugino excited modes in addition to other massless fields, KK zero modes, and boundary fields. The soft terms for the latter massless fields can be extracted from wave-function renormalization [6]. Since there are a large number of KK thresholds between $M_{c}$ and $\Lambda$, the dominant SUSY-breaking effects come from the 
contribution of KK messengers. We will neglect the usual logarithmic contributions in the following. In this approximation, the one-loop renormalization-group equation of the four-dimensional gauge coupling $\alpha$ is [7]

$$
\frac{d \alpha}{d t}=\frac{b_{\mathrm{KK}}}{2 \pi} N(\mu) \alpha^{2},
$$

where $t=\ln \mu$ and $b_{\mathrm{KK}}$ is the beta-function coefficient determined by KK-mode contributions. The energydependent constant $N$ appears in summing up the leading$\log$ contribution and indicates the number of KK states propagating in the loop diagrams. It is approximated by the volume of a sphere with radius $\Lambda / \mu$, which is given by $N(\mu) \simeq \pi^{\delta / 2} / \Gamma(1+\delta / 2)(\Lambda / \mu)^{\delta}$ ( $\delta$ is the number of extra dimensions). The loop-expansion parameter is roughly $N \alpha / 4 \pi$ and is assumed to be small. The gaugino mass is extracted from an auxiliary component of the $T$-dependent renormalized gauge coupling superfield. Integrating Eq. (2), we obtain the soft mass of the gaugino zero mode at the compactification scale,

$$
M_{g}=b_{\mathrm{KK}} N \frac{\alpha}{4 \pi} \frac{F_{T}}{M_{c}},
$$

where $N \equiv N\left(M_{c}\right)$. This result is exact at one loop and not modified even when one considers other bulk interactions of KK states like Yukawa couplings. As is well known, the ratio $M_{g} / \alpha$ is renormalization-group invariant, and therefore Eq. (3) still holds below the compactification scale. Moreover, the ratio is renormalization-group invariant under beta functions due to KK modes [8]. Similar to gauge mediation, $M_{g}$ is proportional to the gauge beta-function coefficient of messenger fields. Our scenario is also similar to the moduli-dominated SUSY breaking in superstring theory [9] where, for the vanishing cosmological constant, soft terms are generated at loop level (string corrections). In particular, the gauge kinetic function has $T$-dependent threshold corrections due to KK modes. In this case, the coefficient is written by the $N=2$ sector and the Green-Schwarz coefficient due to the target-space duality anomaly [10]. Furthermore, it has been shown that the $T$-dependent threshold corrections correspond to the power-law behavior of the gauge coupling constant (2) in a certain limit [11]. On the other hand, in a scenario where bulk gauge fields directly couple to SUSY-breaking sources, that gives rise to tree-level soft masses for bulk zero modes like the dilaton-dominated SUSY-breaking scenario.

Scalar soft masses are derived from wave-function renormalization of four-dimensional chiral multiplets. For a chiral multiplet $\phi$, the one-loop renormalization-group equation for the wave-function factor $Z_{\phi}$ is

$$
\frac{d \ln Z_{\phi}}{d t}=\frac{c_{\mathrm{KK}}}{2 \pi} N(\mu) \alpha
$$

above the compactification scale. The coefficient $c_{\mathrm{KK}}$ is the quadratic Casimir invariant for the $\phi$ representation
[ $c_{\mathrm{KK}}=4 C_{2}\left(R_{\phi}\right)$ ]. In a similar way to the gaugino mass, by integrating the above renormalization-group equations, we obtain the soft SUSY-breaking mass for the scalar component,

$$
m_{\phi}^{2}=\frac{\delta}{2} c_{\mathrm{KK}} N \frac{\alpha}{4 \pi}\left(\frac{F_{T}}{M_{c}}\right)^{2},
$$

that is positive definite. We see that tachyonic modes generally do not appear in contrast to, for example, anomaly mediation [2]. The soft mass terms are generated at oneloop level and proportional to the number of extra dimensions, $\delta$. These properties result from the fact that the beta functions of four-dimensional effective couplings explicitly depend on the energy scale. The scalar trilinear term is also calculated from the wave-function renormalization,

$$
A_{\phi}=c_{\mathrm{KK}} N \frac{\alpha}{4 \pi} \frac{F_{T}}{M_{c}},
$$

and the trilinear coupling in the Lagrangian is given by the sum of $A_{\phi}$ 's of the fields forming a superpotential coupling $\left[\mathcal{L}=-\sum A_{\phi} \phi \partial_{\phi} W(\phi)\right]$. The trilinear terms do not vanish at the boundary scale because of many KK thresholds. Note that when a $\mu$-term generation mechanism is specified, the corresponding holomorphic scalar soft mass, the so-called $B$ term, is derived with the same form as Eq. (6).

In the present approximation, the SUSY-breaking effects are communicated by the KK gauge multiplets that is flavor blind. All induced SUSY-breaking parameters become diagonal and degenerate in the flavor space, and consequently the flavor-changing neutral currents (FCNC) are completely suppressed at the boundary scale. In a more generic case, however, matter and Higgs fields also propagate in the bulk. The corresponding KK modes and their couplings might give new contributions to the FCNC processes. We will see below that even in this case, there are new solutions to the FCNC problem, which are peculiar to the models with extra dimensions.

With bulk matter and Higgs fields, there are additional contributions to soft terms from the Yukawa couplings between the KK matter and Higgs fields. That is, the radius modulus couplings, which induce KK mass terms, give mass splittings in the KK multiplets at each excited level, and the SUSY-breaking effect is transmitted to the visible sector via the Yukawa couplings. In this case, there are two points to notice. First, KK excited states generally belong to the multiplets of $N=2$ or larger supersymmetry in the four-dimensional effective theory. The radiative corrections from the KK multiplets hence cancel out and soft terms are not generated. For example, for bulk zero modes, the gauge contributions disappear $\left(c_{\mathrm{KK}}=0\right)$ because of the hypermultiplet nonrenormalization theorem in unbroken $N=2$ theories. The second point is how to describe Yukawa couplings among $\mathrm{KK}$ and boundary fields. We here assume that Yukawa couplings between KK excited states arise from combinations of trilinear and some higher-dimensional operators. On the other hand, 
Yukawa couplings involving boundary fields are given by local terms in higher-dimensional space [12]. The soft SUSY-breaking terms induced by Yukawa couplings have the expressions

$$
\begin{aligned}
\Delta m^{2} & =-\frac{\delta}{2} a_{\mathrm{KK}} N \frac{\alpha_{y}}{4 \pi}\left(\frac{F_{T}}{M_{c}}\right)^{2}, \\
\Delta A & =-a_{\mathrm{KK}} N \frac{\alpha_{y}}{4 \pi} \frac{F_{T}}{M_{c}} .
\end{aligned}
$$

Here $\alpha_{y}$ is the Yukawa coupling squared, $\alpha_{y}=y^{2} / 4 \pi$, and the positive coefficient $a_{\mathrm{KK}}$ denotes a multiplicity of the Yukawa coupling in the one-loop anomalous dimension. The effects of matter and Higgs KK messengers (7) are opposite in sign to the gauge contributions (5) and (6), and then substantially change the sparticle spectrum. The gaugino mass is, however, unchanged by the new interactions as noted before. It is interesting that gathering together the above results, the KK-mediated SUSY breaking are described in terms of the gauge beta-function $\beta$ and the anomalous dimensions $\gamma_{i}$,

$$
\begin{aligned}
M_{g} & =\frac{\beta}{2 \alpha} \frac{F_{T}}{M_{c}}, \\
m_{i}^{2} & =-\frac{\delta}{2} \gamma_{i}\left(\frac{F_{T}}{M_{c}}\right)^{2}, \quad A_{i}=-\gamma_{i} \frac{F_{T}}{M_{c}}
\end{aligned}
$$

for boundary fields and KK zero modes. Scalar trilinear couplings have no new $C P$-violating phases. The renormalization-group functions $\beta$ and $\gamma_{i}$ are determined by what types of multiplets live in the bulk and on the four-dimensional brane. In other words, sparticle masses measured in future experiments can restrict the patterns of field configuration in the extra dimensions if the KK mediation is dominant. Note that in our scenario, the messenger interactions communicating SUSY breaking are related to the gauge and Yukawa couplings in the SM, which are experimentally observed. Thus the particle and sparticle mass spectra correlate to each other though supersymmetry is broken.

We now examine our results for the SUSY-breaking terms in several examples. The first example is the model described in Ref. [7], in which the SM gauge and Higgs fields propagate in the bulk while the chiral matter multiplets live on a 3-brane. We calculate the soft terms with the formulas (8) in the approximation with the three gauge couplings and the top Yukawa coupling only. The betafunction coefficients are given by $b_{\mathrm{KK}}=(-6,-3,3 / 5)$ for $\mathrm{SU}(3), \mathrm{SU}(2)$, and $\mathrm{U}(1)$, respectively, and we have the gaugino masses,

$$
M_{3}: M_{2}: M_{1}=10 \alpha_{3}: 5 \alpha_{2}: \alpha_{1} .
$$

This ratio holds independently of the value of the compactification scale $M_{c}$ and also remains unchanged under the renormalization group running below $M_{c}$. On the other hand, we have a variety of the scalar soft masses. First, the Higgs soft masses vanish at the leading order. This is because of the absence of radiative corrections from the KK gauge messengers. One can hence get a natural electroweak symmetry breaking without vacuum instability and fine-tuning of parameters even in the case that running effects on the Higgs masses are small, e.g., in the case of low-scale compactification. From this point of view, it may be preferable that Higgs fields are of higher-dimensional origin. Moreover, if the Higgs $\mu$ term is induced by the Yukawa interaction with a gauge-singlet field like the next-to-minimal supersymmetric standard model, the negative contribution to the Higgs soft masses from the Yukawa coupling will cause the symmetry breaking more naturally.

For the squark and slepton masses, we first find that this setup can resolve the supersymmetric FCNC problem. Since the messenger Yukawa couplings are small for the first two generations, the soft masses are degenerate and the FCNC processes are suppressed. With Eqs. (8), the sfermion masses are roughly on the order of the gaugino masses, but the top Yukawa effect reduces the stop masses. Taking $M_{c}$ near below the GUT scale $\left(\simeq 10^{16} \mathrm{GeV}\right)$ and $\delta=1$, for example, we obtain

$$
\begin{aligned}
m_{Q, u_{1,2}}^{2} & \sim 1.7 m_{Q_{3}}^{2} \sim 4 m_{u_{3}}^{2} \sim m_{d}^{2} \\
& \sim 2 m_{L}^{2} \sim 2.5 m_{e}^{2} \sim 2 M_{3}^{2}
\end{aligned}
$$

at $M_{c}$. The relative ratios change through the renormalization-group evolutions down to low energy. In this model, the lightest SUSY particle is the bino. The bino mass becomes rather small compared to the gluino mass, $M_{3} / M_{1} \sim 60$ at low energy, and that may conflict with the experimental lower bound of $M_{1}$ and the naturalness problem. This problem, however, can easily be avoided, for example, by adding bulk fields charged under the U(1) gauge symmetry. The detailed analyses of spectrum, including the symmetry breaking issue, etc., will be discussed elsewhere. We finally comment that in this model, no-scale supergravitylike boundary conditions $\left(\sqrt{m^{2}}, A \ll M_{g}\right)$ [13] may be viable if a small value of our 3-brane tension would suppress the couplings between bulk and boundary fields [14]. In this case, the flavor-changing operators are also suppressed.

The second example is the case that all the supersymmetric standard model fields propagate in the bulk. If assuming the $\mathrm{KK}$ excited modes consist in $N=4$ supermultiplets (i.e., a finite theory), they have no effects on wave-function renormalization and the running behaviors of couplings become logarithmic. The soft SUSYbreaking terms are then determined by the beta functions of the low-energy theory like the case of anomaly mediation $[2,15]$. On the other hand, if the KK excited modes belong to $N=2$ supermultiplets, the gaugino masses are generated by nonzero gauge beta functions while the scalar soft terms vanish at one-loop level. We thus find a no-scale supergravity boundary condition for the soft terms of the 
low-energy fields. In this example, the gaugino masses become

$$
M_{3}: M_{2}: M_{1}=6 \alpha_{3}: 9 \alpha_{2}: \frac{63}{5} \alpha_{1} \text {. }
$$

The relation also holds at low energy. The other soft terms are generated by the gaugino masses via renormalizationgroup running down to low energy.

This example shows a new possibility for solving the supersymmetric FCNC problem. As seen from (8), bulk zero modes generally have suppressed soft scalar masses compared to those of boundary fields as well as gaugino masses. This is due to the restricted vertex structure (nonrenormalization property) of higher supersymmetry on the KK excited states. Even when flavor-changing interactions communicate SUSY breaking, their contributions to soft terms vanish for KK zero modes, and the FCNC processes involving these fields are suppressed. Note that the situation is in contrast to the recently proposed gauginomediation scenario [3]. There, bulk fields couple to the SUSY-breaking source on a hidden brane and obtain soft masses for zero modes. As a result, matter multiplets are obliged to exist only in the visible sector to suppress the dangerous FCNC processes.

We finally comment on the gravitino mass scale. If we assume $F_{T}$ is the only SUSY-breaking source, the gravitino mass $m_{3 / 2}$ is estimated by requiring a vanishing effective cosmological constant, as $m_{3 / 2} \simeq F_{T} / M_{\mathrm{pl}} K(T)$. Here $K$ is the function of Kähler potential and $T$. Since we now consider $M_{c}<M_{\mathrm{pl}}$, the gravitino becomes lighter than other SUSY particles and may be the lightest one. That gives a cosmological bound for $m_{3 / 2}[<O(\mathrm{keV})]$, and then for the magnitude of $K$. (New inflation scenario could avoid this bound with a low reheating temperature [16].) For example, assuming the no-scale type Kähler form, $K \sim T+T^{*}$, it leads an upper bound of the compactification scale; $M_{c} \lesssim 10^{-(2-3)} M_{\mathrm{pl}} \sim 10^{16} \mathrm{GeV}$.

In summary, we have shown that the nonvanishing $F$ term of the radius modulus gives a new mediation mechanism for SUSY breaking. There, KK excited states transmit the breaking effects to the visible sector via the gauge and Yukawa couplings, which are experimentally observed. The soft SUSY-breaking parameters are expressed in terms of wave-function factors, and the resulting spectrum is shown to have rich structure depending on how the SM fields extend in the bulk. The FCNC problem is solved with the renormalization property of higher supersymmetry or a finite width of 3-brane, both of which can suppress the KK-mode couplings. Our SUSY mediation mechanism also gives a framework of natural electroweak symmetry breaking. It is noted that the spectrum is in contrast to the case that there is a SUSY-breaking source somewhere in the extra dimensions and bulk fields directly couple to it. In that case, the bulk zero modes obtain sizable soft scalar masses, which are generally nonuniversal, but boundary fields have suppressed ones.

The authors thank T. Hirayama, K. Kohri, J. Kubo, Y. Nomura, S. Sugimoto, and M. B. Tachibana for helpful discussions and comments.

Note added.-Similar ideas of using the $F$ component of the radius modulus is discussed in Ref. [17].

[1] S. Dimopoulos and H. Georgi, Nucl. Phys. B193, 150 (1981); J. Ellis and D. V. Nanopoulos, Phys. Lett. 110B, 44 (1981).

[2] L. Randall and R. Sundrum, Nucl. Phys. B557, 79 (1999).

[3] D. E. Kaplan, G. D. Kribs, and M. Schmaltz, Phys. Rev. D 62, 035010 (2000); Z. Chacko, M. A. Luty, A.E. Nelson, and E. Ponton, J. High Energy Phys. 0001, 003 (2000).

[4] I. Antoniadis, Phys. Lett. B 246, 377 (1990); I. Antoniadis, S. Dimopoulos, A. Pomarol, and M. Quiros, Nucl. Phys. B544, 503 (1999); M. Sakamoto, M. Tachibana, and K. Takenaga, Phys. Lett. B 458, 231 (1999); N. Arkani-Hamed, L. Hall, D. Smith, and N. Weiner, hep-ph/9911421.

[5] M. A. Luty and R. Sundrum, Phys. Rev. D 62, 035008 (2000).

[6] G. F. Giudice and R. Rattazzi, Nucl. Phys. B511, 25 (1998).

[7] K. R. Dienes, E. Dudas, and T. Gherghetta, Phys. Lett. B 436, 55 (1998); Nucl. Phys. B537, 47 (1999).

[8] T. Kobayashi, J. Kubo, M. Mondragon, and G. Zoupanos, Nucl. Phys. B550, 99 (1999).

[9] L. E. Ibáñez and D. Lüst, Nucl. Phys. B382, 305 (1992); B. de Carlos, J.A. Casas, and C. Muñoz, Phys. Lett. B 299, 234 (1993); A. Brignole, L. E. Ibáñez, and C. Muñoz, Nucl. Phys. B422, 125 (1994); T. Kobayashi, D. Suematsu, K. Yamada, and Y. Yamagishi, Phys. Lett. B 348, 402 (1995).

[10] J.-P. Derandinger, S. Ferrara, C. Kounnas, and F. Zwirner, Nucl. Phys. B372, 145 (1992).

[11] D. Ghilencea and G. Ross, Nucl. Phys. B569, 391 (2000).

[12] E. A. Mirabelli and M.E. Peskin, Phys. Rev. D 58, 065002 (1998).

[13] For a review, A. B. Lahanas and D. V. Nanopoulos, Phys. Rep. 145, 1 (1987).

[14] M. Bando, T. Kugo, T. Noguchi, and K. Yoshioka, Phys. Rev. Lett. 83, 3601 (1999).

[15] G. F. Giudice, M. A. Luty, H. Murayama, and R. Rattazzi, J. High Energy Phys. 9812, 027 (1998).

[16] J. Ellis, G. B. Gelmini, J. L. Lopez, D. V. Nanopoulos, and S. Sarkar, Nucl. Phys. B373, 399 (1992); E. Holtmann, M. Kawasaki, K. Kohri, and T. Moroi, Phys. Rev. D 60, 023506 (1999).

[17] Z. Chacko and M. A. Luty, hep-ph/0008103. 